\title{
Estudo Numérico de Caminhos Altamente Condutivos em Forma de Y para Arrefecimento de Corpos com Geração de Calor
}

Cássia C. Beckel ${ }^{1}$

Programa de Pós-Graduação em Engenharia Mecânica, UFRGS, Porto Alegre, RS

Luiz A. O.Rocha ${ }^{2}$

Departamento de Engenharia Mecânica, UFRGS, Porto Alegre, RS

Liércio A. Isoldi ${ }^{3}$

Escola de Engenharia, FURG, Rio Grande, RS

Elizaldo D. dos Santos ${ }^{4}$

Escola de Engenharia, FURG, Rio Grande, RS

Resumo. Este estudo numérico utiliza o método Design Construtal para reduzir os pontos quentes de um sistema com geração de calor uniforme por unidade de volume por condução. A ideia é facilitar o acesso do fluxo de calor através de vias de alta condutividade em forma de $\mathrm{Y}$ e a sua liberação para o ambiente. O objetivo é o de minimizar a máxima temperatura em excesso adimensional de todo o sistema (materiais de condutividade alta e baixa). A forma do sistema pode variar sujeita à duas restrições: o volume total e o volume das vias de alta condutividade. Os resultados mostram que, para o caso estudado a geometria tende para uma forma de V (isto é o tronco do corpo, sem geração de calor tende a diminuir na direção do dissipador de calor). Os resultados também demonstram a aplicabilidade do Design Construtal para a melhoria do desempenho térmico do sistema, por exemplo, a otimização de dois graus de liberdade ocasiona uma diminuição de $25 \%$ na máxima temperatura em excesso adimensional da melhor configuração quando ela é comparada com a pior configuração estudada.

Palavras-chave. Design Construtal, Geração de calor, Vias em forma de Y.

\section{Introdução}

Vários estudos tem sido realizados a fim de diminuir a temperatura de uma superfície, onde há geração de calor, utilizando-se apenas o mecanismo de difusão de calor. Arrefecimento de eletrônicos (resistores, transistores, diodos e capacitores), cujo objetivo é o de minimizar a temperatura máxima num dado volume finito considerando o calor gerado em cada ponto, têm sido amplamente estudados na literatura por [1-3].

\footnotetext{
${ }^{1}$ cassia.beckel@ufrgs.br

${ }^{2}$ luizrocha@mecanica.ufrgs.br

${ }^{3}$ liercioisoldi@furg.br

${ }^{4}$ elizaldosantos@furg.br
} 
A lei Construtal pode ser concebida como uma generalização de uma tendência de todas as coisas fluírem ao longo de caminhos de resistência mínima: natureza e engenharia estão unidas na busca de uma melhor arquitetura fluxo. Esta lei tem sido utilizada para diversas aplicações em todos os domínios da geração de design e evolução, da biologia e física a organização social, a evolução da tecnologia, sustentabilidade e engenharia [4-6].

O primeiro trabalho nesta área foi apresentado por [7] que desenvolveu um design de arrefecimento constituído por uma placa quadrada com um material de condutividade térmica elevada inserido no seu centro. Fixando as áreas da placa e as vias de elevada condutividade, as dimensões foram otimizadas, a fim de conseguir a minimização da resistência térmica não-dimensional. Designs de vias de alta condutividade foram testados para outras geometrias, tais como as configurações em forma de X, Y e I [8-10], na literatura, concluindo que os mesmos princípios físicos encontrados na natureza para a determinação da forma e estrutura em sistemas de fluxo animados e inanimados podem ser empregados na engenharia para alcançar o melhor desempenho térmico.

Portanto, o presente trabalho destina-se a descobrir a configuração que facilita o fluxo de calor por condução a partir de uma placa com geração de calor para o ambiente, utilizando um caminho de alta condutividade térmica em forma de Y ligado a um ponto de baixa temperatura (dissipador de calor). Assume-se que o volume elementar quadrado que gera calor uniformemente por unidade de volume é arrefecido por um dissipador de calor à temperatura $T_{0}$ que está localizado na borda do sistema. O estudo realizado expande as configurações estudadas por [10].

\section{Modelo Matemático}

Considere o corpo condutivo mostrado na Figura 1. Por simplificação, a configuração utilizada é bidimensional, com a terceira dimensão $(W)$ suficientemente longa em comparação com a altura $(H)$ e o comprimento $(L)$ do volume total. São inseridos caminhos condutivos em forma de $\mathrm{Y}$ compostos por um material de alta condutividade térmica $\left(k_{p}\right)$ na base e nos ramos e na região definida pelo trapézio, inseridos no corpo com condutividade térmica inferior $(k)$.

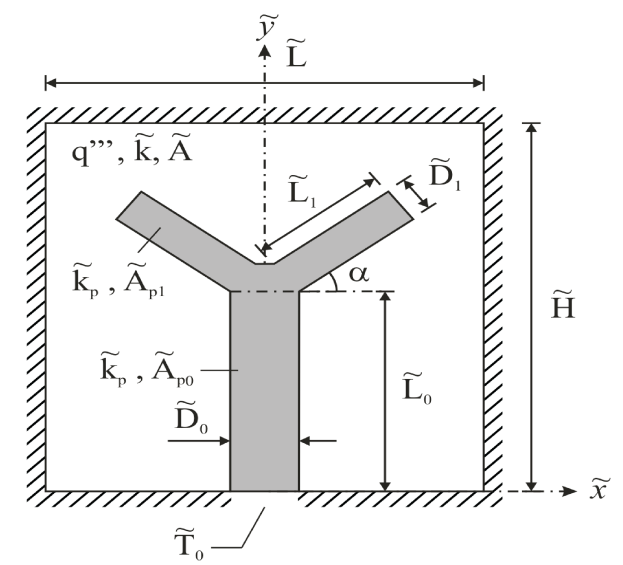

Figura 1: Áreas do domínio e as principais dimensões do corpo altamente condutor a ser analisado. 
A corrente de calor gerada $q^{\prime \prime \prime}(A W)$ é removida pelos dissipadores de calor localizados na borda do corpo à temperatura $T_{0}$. O trabalho consiste em calcular a máxima temperatura em excesso adimensional $\left(T_{\max }-T_{0}\right) /\left(q^{\prime \prime \prime} A / k\right)$ e avaliar como a geometria $\left(L_{1} / L_{0}\right.$ e $\left.\alpha\right)$ influencia na remoção da corrente de calor. Para isso, de acordo com o Design Construtal, duas restrições devem ser consideradas. São elas: a restrição da área total:

$$
A=H L
$$

e a restrição da área ocupada pelos materiais de alta condutividade:

$$
A_{p}=L_{0} D_{0}+\left(\frac{D_{0}+\left(D_{0}-2 X\right)}{2}\right) Y+2 L_{1} D_{1}
$$

Na Equação (2), o termo $\left(D_{0}-2 X\right)$ representa a linha superior do trapézio, que liga a haste e os ramos bifurcados (linha tracejada na Figura 1) e a variável "Y" é a altura do trapézio. Os termos que surgem na Equação (2) são dados por:

$$
\begin{aligned}
& X=D_{1} \cos (\beta) \\
& Y=D_{1} \sin (\beta)
\end{aligned}
$$

onde:

$$
\beta=\frac{\pi}{2}-\alpha
$$

A razão entre as Equações (1) e (2) pode ser expressada pela fração de área $\phi$ :

$$
\phi=\frac{A_{p}}{A}
$$

A análise que proporciona a máxima temperatura em excesso adimensional como uma função da geometria consiste em solucionar numericamente a equação da condução de calor para a região com geração de calor e baixa condutividade $k$ :

$$
\frac{\partial^{2} \theta}{\partial \tilde{x}^{2}}+\frac{\partial^{2} \theta}{\partial \tilde{y}^{2}}+1=0
$$

e para a região sem geração de calor e alta condutividade térmica:

$$
\frac{\partial^{2} \theta}{\partial \tilde{x}^{2}}+\frac{\partial^{2} \theta}{\partial \tilde{y}^{2}}=0
$$

As variáveis adimensionais do problema são:

$$
\tilde{x}, \tilde{y}, \tilde{H}, \tilde{L}, \tilde{L}_{0}, \tilde{D}_{0}, \tilde{L}_{1}, \tilde{D}_{1}=\frac{x, y, H, L, L_{0}, D_{0}, L_{1}, D_{1}}{A^{1 / 2}}, \quad \tilde{k}_{p}, \tilde{k}=\frac{\left(k_{p}, k\right)}{k}
$$

As superfícies exteriores são adiabáticas e suas condições de contorno são:

$$
\frac{\partial \theta}{\partial \tilde{n}}=0
$$


onde $\tilde{n}$ são as superfícies isoladas. A condição de contorno na região $\left(-\tilde{D}_{0} / 2 \leq \tilde{x} \leq\right.$ $\left.\tilde{D}_{0} / 2 ; \tilde{y}=0\right)$ que está em contato com o dissipador de calor é dada por:

$$
\theta_{0}=0
$$

Já a máxima temperatura em excesso adimensional, $\theta_{\max }$, é definida por:

$$
\theta_{\max }=\frac{T_{\max }-T_{0}}{q^{\prime \prime \prime} A / k}
$$

Com o problema totalmente formulado e a geometria definida, a máxima temperatura em excesso adimensional pode ser calculada com os parâmetros estabelecidos, variando os graus de liberdade estabelecidos para o problema.

\subsection{Modelo Numérico}

A função definida pela Equação (12) pode ser determinada através da solução numérica das equações de condução de calor (7) e (8) para o campo de temperatura em cada configuração assumida dependendo dos graus de liberdade estabelecidos. A malha apropriada é determinada através de sucessivos refinamentos, aumentando o número de elementos até que o critério $\left|\left(\theta_{\max }^{j}-\theta_{\max }^{j+1}\right) / \theta_{\max }^{j}\right|<1,0 \times 10^{-4}$, onde $\theta_{\max }^{j}$ representa a máxima temperatura em excesso adimensional calculada com base na malha atual gerada e $\theta_{\max }^{j+1}$ corresponde à máxima temperatura em excesso adimensional obtida considerando a malha gerada após o refinamento, onde o número de elementos triangulares é incrementado.

Para determinar a solução numérica das Equações (7) e (8) é utilizado um código de elementos finitos, baseado em elementos triangulares, desenvolvido em ambiente MatLabß [11], mais precisamente no PDE (partial-differential-equations) Toolbox. A Tabela 1 mostra como a independência de malha foi obtida. A malha independente para o problema, mostrada na Tabela 1 é composta por 138.624 elementos triangulares.

Tabela 1: Teste numérico a malha independente do número de elementos $(\phi=0,2, H / L=$ $1,0, \quad \tilde{D}_{0}=0,7, L_{1} / L_{0}=143, D_{1} / D_{0}=0,14, \tilde{k}_{p}=300$ e $\left.\alpha=89^{\circ}\right)$.

\begin{tabular}{|c|c|c|}
\hline Número de elementos & $\theta_{\text {max }}^{j}$ & $\left|\left(\theta_{\max }^{j}-\theta_{\max }^{j+1}\right) / \theta_{\max }^{j}\right|$ \\
\hline 2.166 & 0,041082 & $3,1803 \times 10^{-3}$ \\
\hline 8.664 & 0,041213 & $9,4072 \times 10^{-4}$ \\
\hline 34.656 & 0,041252 & $2,6554 \times 10^{-4}$ \\
\hline $138.624^{(1)}$ & 0,041263 & $7,3607 \times 10^{-5}$ \\
\hline 554.496 & 0,041266 & - \\
\hline
\end{tabular}

(1) malha independente

\section{Resultados}

No presente estudo, foi realizada uma avaliação do efeito da razão de aspecto $L_{1} / L_{0} \mathrm{e}$ do ângulo $\alpha$ na máxima temperatura em excesso adimensional, $\theta_{\max }$ no domínio mostrado 
na Figura 1. Além disso, um processo de otimização geométrica foi aplicado através do método de busca exaustiva, ou seja, foram testadas todas a geometrias possíveis propostas pelo Design Construtal. O processo de otimização foi dividido em duas etapas. No primeiro passo, a geometria é otimizada variando a razão de aspecto $L_{1} / L_{0}$ e mantendo fixos os três parâmetros geométricos: $\left(\alpha, D_{1} / D_{0}\right.$ e $\left.\tilde{D}_{0}\right)$. Assim, a máxima temperatura em excesso adimensional é uma vez minimizada, $\theta_{\max , m}$ e a correspondente razão de aspecto ótima $L_{1} / L_{0}$ também é minimizada, $\left(L_{1} / L_{0}\right)_{o}$. E na segunda etapa, o mesmo processo foi repetido para diferentes valores de $\alpha$ e mantendo-se fixos os dois parâmetros geométricos $\left(D_{1} / D_{0}\right.$ e $\left.\tilde{D}_{0}\right)$. Dessa forma, tanto a máxima temperatura em excesso adimensional quanto a razão de aspecto $L_{1} / L_{0}$ foram minimizadas duas vezes, ou seja, foram obtidos $\theta_{\max , m m}$ e $\left(L_{1} / L_{0}\right)_{o o}$. Já o ângulo $\alpha$ foi minimizado apenas uma vez, $\alpha_{o}$.

Inicialmente, foram assumidos os seguintes parâmetros para a simulação: $\phi=0,2, \tilde{k}_{p}=$ $300, \alpha=60^{\circ}, 70^{\circ}$ e $80^{\circ}, \tilde{D}_{0}=0,29, D_{1} / D_{0}=0,36$. A Figura 2 mostra que existe uma razão de aspecto ótima que minimiza máxima temperatura em excesso adimensional para cada $\alpha$ estudado.

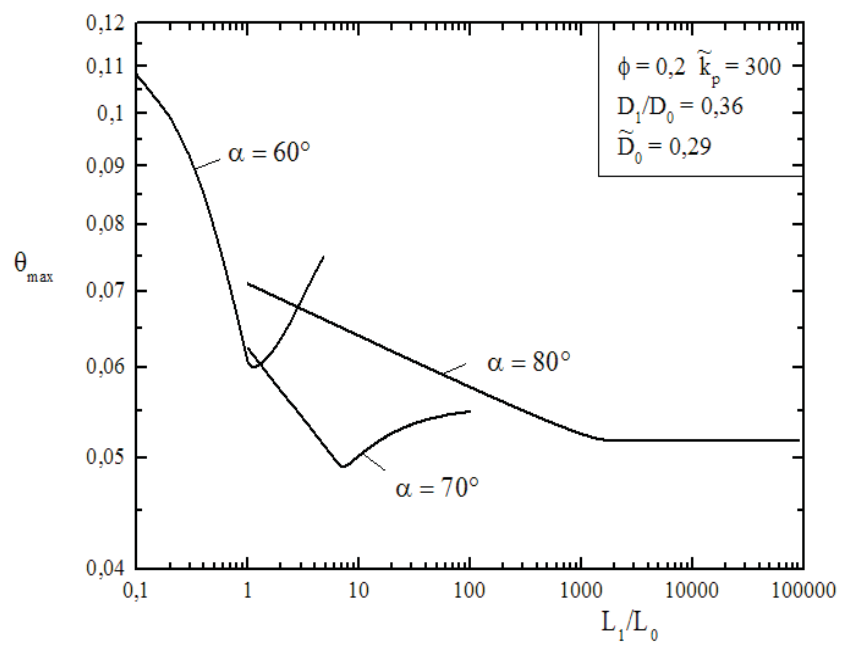

Figura 2: Primeira otimização: efeito da razão de aspecto $L_{1} / L_{0}$.

O processo utilizado para obter os resultados mostrados na Figura 2 foi repetido para diferentes valores de $\alpha$ e variando a razão de aspecto $\left(L_{1} / L_{0}\right)_{o}$. A máxima temperatura em excesso adimensional $\theta_{\max , m}$ obtida na segunda etapa do processo de otimização, variando $\left(L_{1} / L_{0}\right)_{o}$, é mostrada na Figura 3. O melhor desempenho foi obtido na região definida entre $71^{\circ} \leq \alpha \leq 75^{\circ}$ e o valor ótimo ocorre em $\alpha_{o}=73^{\circ}$, dado que o subscrito "o" indica que o ângulo foi otimizado uma vez. A Figura 3 mostra que existe uma relação ótima $\left(L_{1} / L_{0}\right)_{o o}=75,1$ que minimiza a máxima temperatura em excesso adimensional, $\theta_{\max , m m}=0,044914 \mathrm{em} \alpha_{o}=73^{\circ}$. A configuração ótima, com $\alpha_{o}=73^{\circ}$ é comparada com a pior configuração $\left(\alpha=60^{\circ}\right)$ e resulta em uma diminuição de aproximadamente $25 \%$ na máxima temperatura em excesso adimensional.

A configuração que mais facilita o fluxo de calor obtida na Figura 3 para $\alpha=73^{\circ}$ é 


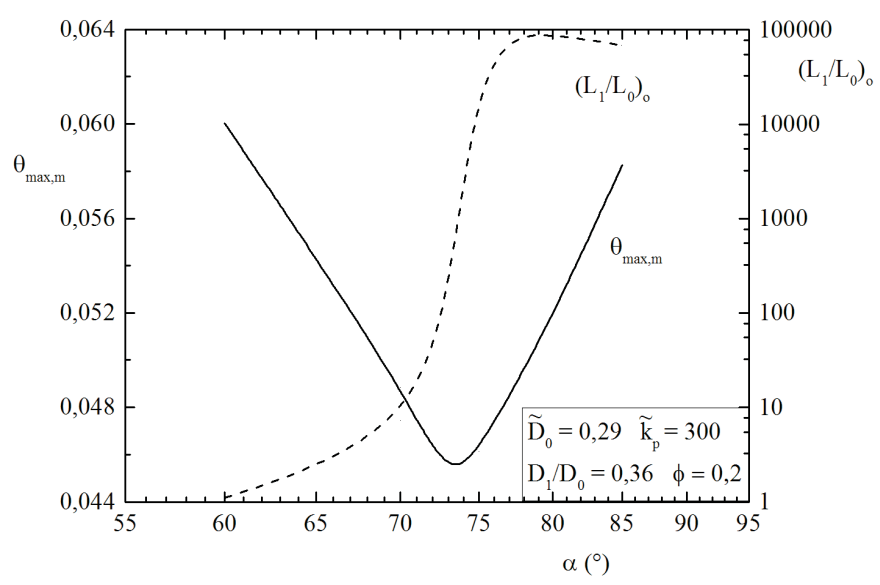

Figura 3: Segunda otimização: efeito do ângulo $\alpha$.

mostrada na Figura 4(b).

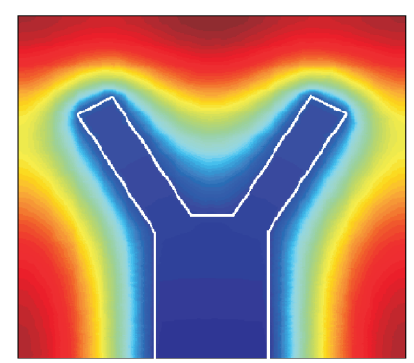

(a)

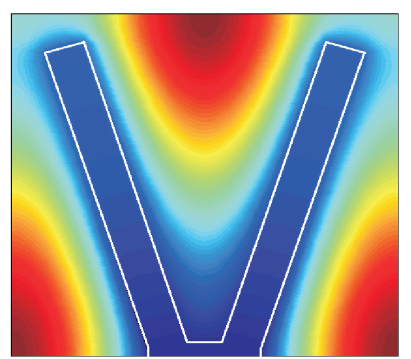

(b)

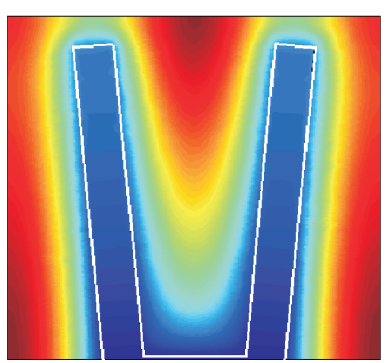

(c)

Figura 4: a) $\alpha=60^{\circ}, L_{1} / L_{0}=1,1$ e $\theta_{\max }=0,060025 \quad$ b) $\alpha=73^{\circ}, L_{1} / L_{0}=75,1$ e $\theta_{\max }=$ 0,044914 c) $\alpha=85^{\circ}, L_{1} / L_{0}=67000$ e $\theta_{\max }=0,058253$.

Nota-se que a configuração mostrada na Figura 4(b) redistribui as imperfeições, isto é, os pontos quentes, de acordo com o princípio da ótima distribuição das imperfeições conforme o princípio Construtal [4]. As Figuras 4(a) e 4(c) apresentam outras configurações otimizadas somente para o grau de liberdade $L_{1} / L_{0}$.

\section{Conclusões}

Este trabalho aplicou o método Design Construtal para obter a arquitetura que maximize o desempenho das vias em forma de $\mathrm{Y}$ de um material de alta condutividade incorporado no corpo de menor condutividade que gera calor uniformemente. A análise realizada minimizou duas vezes a máxima temperatura em excesso adimensional, considerando os dois graus de liberdade definidos para a configuração, $L_{1} / L_{0}$ e $\alpha$, para uma fração de área constante, $\phi=0,2$ e $k_{p}=300$. Os resultados apontam que $\alpha$ tem um 
grande efeito sobre $\theta_{\max , m m}$, isto é, a configuração com $\alpha=73^{\circ}$ tem um desempenho $25 \%$ melhor quando comparado com a configuração em que $\alpha=60^{\circ}$ que é a configuração que tem o pior desempenho entre as configurações otimizadas uma vez. Os resultados também mostram que o Design Construtal é um método confiável na busca de configurações ótimas que facilitam o fluxo de calor.

\section{Agradecimentos}

Luiz A. O. Rocha, Liércio A. Isoldi, Elizaldo D. dos Santos e Cássia C. Beckel agradecem o suporte financeiro do CNPq, Brasília, DF.

\section{Referências}

[1] A. Bejan. Shape and Structure, from Engineering to Nature. Cambridge University Press, Cambridge, 2000.

[2] R. Boichot, L. Luo, and Y. Fan. Tree-network structure generation for heat conduction by cellular automaton, Journal Energy Conversion and Management, 50:376-386, 2019 .

[3] Y. A. Çengel and A. J. Ghajar. Heat \& Mass Transfer: A Practical Approach. MC Graw-Hill, New York, 2012.

[4] A. Bejan., and S. Lorente. Design with Constructal Theory. John Wiley \& Sons, New Jersey, 2008.

[5] A. Bejan., and S. Lorente. Constructal law of design and evolution: Physics, biology, technology, and society, Journal of Applied Physics, 113:301-321, 2013.

[6] A. Bejan., and J. P. Zane. Design in Nature. Doubleday, New York., 20012.

[7] A. Bejan. Constructal-theory network of conducting paths for cooling a heat generating volume, Journal Heat Mass Transfer, 40:799-816, 1997.

[8] G. Lorenzini, C. Biserni, and L.A.O. Rocha. Constructal design of X-shaped conductive pathways for cooling, Int. Journal Heat Mass Transfer, 58:513-520, 2013.

[9] G. Lorenzini, C. Biserni, and L.A.O. Rocha. Constructal design of non-uniform Xshaped conductive pathways for cooling a heat-generating body, Int. Journal of Themral Sciences, 71:140-147, 2013.

[10] C.S. Horbach, E.D.Dos Santos, L.A. Isoldi, and L.A.O. Rocha. Constructal design of Y-shaped pathways for cooling a heat-generating body, Defect and Diffusion Forum, 348:245-260, 2014.

[11] MatLab. User's Guide, Version 6.0.088, Release 12. The Mathworks Inc., 2000. 